\title{
Novel Tap-wise LMMSE Channel Estimation for MIMO W-CDMA
}

\author{
Christian Mehlführer and Markus Rupp \\ Institute of Communications and Radio-Frequency Engineering \\ Vienna University of Technology \\ Gusshausstrasse 25/389, A-1040 Vienna, Austria \\ Email: \{chmehl, mrupp $\} @$ nt.tuwien.ac.at \\ Web: http://www.nt.tuwien.ac.at/rapid-prototyping
}

\begin{abstract}
In this paper, a tap-wise LMMSE channel estimator for MIMO W-CDMA is derived. Descrambling operations applied to delayed versions of the received signal whiten the input signal. The descrambling process thus breaks up the full channel autocorrelation matrix (including spatial and temporal correlation) into several independent, small autocorrelation matrices that include only spatial correlation. The problem of estimating the channel autocorrelation matrices is tackled by an "instantaneous" autocorrelation estimator that uses only one channel estimate (obtained by a very low complexity channel estimator) as input. Simulation results of a MIMO HSDPA system show that the tap-wise LMMSE estimator with instantaneous channel autocorrelation estimation yields performance gains of up to $0.85 \mathrm{~dB}$ over a correlation-based channel estimator. Even larger gains can be achieved with improved channel autocorrelation estimators.
\end{abstract}

\section{INTRODUCTION}

Channel estimation in W-CDMA networks is strongly affected by interference that can be divided into intra-cell and inter-cell interference. At the basestation usually only a small amount of power (approx. 10\%) is dedicated to the pilot channels that can be used for channel estimation. The remaining power is dedicated to all other channels and is thus considered as intra-cell interference for the channel estimator. The amount of intra-cell interference increases with the number of users receiving data in one cell since more spreading codes are used simultaneously at the basestation. Furthermore, the intracell interference also increases with the number of transmit antennas (which becomes for example relevant for the already specified MIMO extension in UMTS [1]) at the basestation since the same spreading codes are reused at each transmit antenna. Inter-cell interference becomes crucial at the cell edges where the received power of the desired basestation is in the order of the received power from other basestations.

A good channel estimator performance can therefore only be achieved if the estimator takes all types of interference into account. This can be done for example by an LMMSE channel estimator. However, it turns out that the resulting matrices that have to be inverted become very huge $(5120 \times 5120$ for a $2 \times 2$ MIMO system with slot based channel estimation) [2] prohibiting real-time implementations. In this paper we propose a so-called tap-wise LMMSE channel estimator that whitens the receive signal by a descrambling operation. It is shown in the paper that the descrambling operation removes the temporal correlation from the received signal. Thus, the descrambling allows for tap-wise channel estimation which is of very low complexity.

The paper is organized as follows. Section II describes our system model which considers intra and inter-cell interference. Three kinds of channel estimators, namely the LS estimator, a correlation-based estimator, and the tap-wise LMMSE estimator, are presented in Section III. Simulation results utilizing a physical layer TxAA (Transmit Antenna Array) [3, 4] HSDPA (High Speed Downlink Packet Access) simulator are presented in Section IV. Finally, we draw our conclusions in Section V.

\section{System MOdEL}

We define the spread pilot chip sequence $\mathbf{p}_{i}^{\left(n_{\mathrm{t}}\right)}$ and the spread (data + control + synchronization) sequences $\mathbf{d}_{i}^{\left(n_{\mathrm{t}}\right)}$ that have to be transmitted at antenna $n_{\mathrm{t}}$ as

$$
\begin{aligned}
& \mathbf{p}_{i}^{\left(n_{\mathrm{t}}\right)}=\left[p_{i}^{\left(n_{\mathrm{t}}\right)}, \ldots, p_{i+N_{\mathrm{c}}-1}^{\left(n_{\mathrm{t}}\right)}\right]^{\mathrm{T}}, \\
& \mathbf{d}_{i}^{\left(n_{\mathrm{t}}\right)}=\left[d_{i}^{\left(n_{\mathrm{t}}\right)}, \ldots, d_{i+N_{\mathrm{c}}-1}^{\left(n_{\mathrm{t}}\right)}\right]^{\mathrm{T}},
\end{aligned}
$$

where $i$ is the time index in chips and $N_{\mathrm{c}}$ is the number of chips considered for the channel estimation. We assume that the channel stays constant during the transmission of these $N_{\mathrm{c}}$ chips. The scrambled transmit signal at antenna $n_{\mathrm{t}}$ is given by

$$
\mathbf{x}_{i}^{\left(n_{\mathrm{t}}\right)}=\mathbf{S}_{i}\left(\mathbf{p}_{i}^{\left(n_{\mathrm{t}}\right)}+\mathbf{d}_{i}^{\left(n_{\mathrm{t}}\right)}\right) \quad ; n_{\mathrm{t}}=1 \ldots N_{\mathrm{T}}
$$

with the matrix $\mathbf{S}_{i}=\operatorname{diag}\left[s_{i}, \ldots, s_{i+N_{\mathrm{c}}-1}\right]$ comprising $N_{\mathrm{c}}$ chips of the base station's scrambling sequence of the at the main diagonal. The combined transmit signal of all $N_{\mathrm{T}}$ transmit antennas can be modeled by horizontally stacking the transmit sequences of the individual antennas:

$$
\begin{aligned}
\mathbf{P}_{i} & =\left[\mathbf{p}_{i}^{(1)}, \ldots, \mathbf{p}_{i}^{\left(N_{\mathrm{T}}\right)}\right], \\
\mathbf{D}_{i} & =\left[\mathbf{d}_{i}^{(1)}, \ldots, \mathbf{d}_{i}^{\left(N_{\mathrm{T}}\right)}\right], \\
\mathbf{X}_{i} & =\left[\mathbf{x}_{i}^{(1)}, \ldots, \mathbf{x}_{i}^{\left(N_{\mathrm{T}}\right)}\right]=\mathbf{S}_{i}\left(\mathbf{P}_{i}+\mathbf{D}_{i}\right) .
\end{aligned}
$$


The $N_{\mathrm{T}} \times N_{\mathrm{R}}$ MIMO channel matrix at the delay $k(k=$ $\left.0, \ldots, L_{\mathrm{h}}-1\right)$ is defined as

$$
\mathbf{H}_{k}=\left[\begin{array}{ccc}
h_{k}^{(1,1)} & \ldots & h_{k}^{\left(1, N_{\mathrm{R}}\right)} \\
\vdots & \ddots & \vdots \\
h_{k}^{\left(N_{\mathrm{T}}, 1\right)} & \ldots & h_{k}^{\left(N_{\mathrm{T}}, N_{\mathrm{R}}\right)}
\end{array}\right]
$$

Using the definitions of the transmit signal $\mathbf{X}_{i}$ and of the channel matrices $\mathbf{H}_{k}$, we can express the receive signal of all receive antennas compactly as

$$
\begin{aligned}
\mathbf{Y}_{i} & =\left[\mathbf{y}_{i}^{(1)}, \ldots, \mathbf{y}_{i}^{\left(N_{\mathrm{R}}\right)}\right]= \\
& =\sum_{k=0}^{L_{\mathrm{h}}-1} \mathbf{S}_{i-k}\left(\mathbf{P}_{i-k}+\mathbf{D}_{i-k}\right) \mathbf{H}_{k}+\mathbf{N}_{i}
\end{aligned}
$$

Here, the matrix $\mathbf{N}_{i}$ accounts for the interference of other basestations (inter-cell interference), as well as the thermal noise generated in the radio frequency frontend of the receiver. Note that the actual structure of $\mathbf{N}_{i}$ is irrelevant for the tapwise LMMSE estimator since the interference will be whitened by the descrambling operations.

\section{Channel ESTIMATORS}

In this section, we derive the Least Squares and a correlation-based channel estimator. These estimators are often used in CDMA systems because of their low complexity[5]. Furthermore, we derive the tap-wise LMMSE estimator that shows superior performance compared to the two other estimators.

\section{A. Least Squares Estimator}

A low complexity channel estimator with reasonable performance is the Least Squares (LS) channel estimator. The LS estimator can be derived by reformulating the system description in Equation (6) to

$$
\mathbf{Y}_{i}=\tilde{\mathbf{P}}_{i} \mathbf{H}+\tilde{\mathbf{N}}_{i}
$$

where we used the stacked matrices

$$
\begin{gathered}
\tilde{\mathbf{P}}_{i}=\left[\mathbf{S}_{i} \mathbf{P}_{i}, \mathbf{S}_{i-1} \mathbf{P}_{i-1}, \ldots, \mathbf{S}_{i-L_{\mathrm{h}}+1} \mathbf{P}_{i-L_{\mathrm{h}}+1}\right], \\
\mathbf{H}=\left[\mathbf{H}_{0}^{\mathrm{T}}, \ldots, \mathbf{H}_{L_{\mathrm{h}}-1}^{\mathrm{T}}\right]^{\mathrm{T}}
\end{gathered}
$$

and

$$
\tilde{\mathbf{N}}_{i}=\sum_{k=0}^{L_{\mathrm{h}}-1} \mathbf{S}_{i-k} \mathbf{D}_{i-k} \mathbf{H}_{k}+\mathbf{N}_{i}
$$

It can be easily verified that the LS estimator for the system description (7) is given by

$$
\hat{\mathbf{H}}^{(\mathrm{LS})}=\left(\tilde{\mathbf{P}}_{i}^{\mathrm{H}} \tilde{\mathbf{P}}_{i}\right)^{-1} \tilde{\mathbf{P}}_{i}^{\mathrm{H}} \mathbf{Y}_{i}
$$

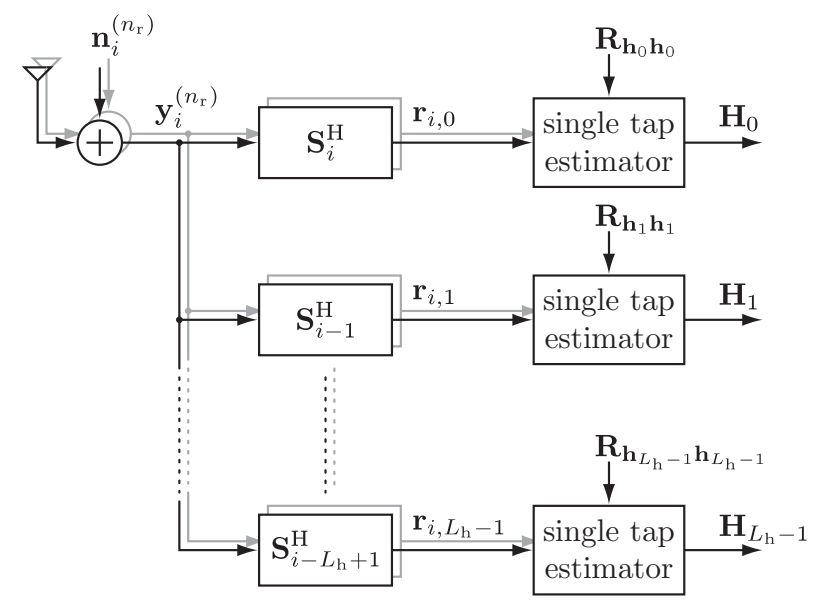

Fig. 1. Tap-wise LMMSE channel estimator.

\section{B. Correlation-based Estimator}

The matrix $\left(\tilde{\mathbf{P}}_{i}^{\mathrm{H}} \tilde{\mathbf{P}}_{i}\right)$ that has to be inverted during the calculation of the LS channel estimate is close to a diagonal matrix. Therefore, a good approximation for (11) is given by [6]

$$
\hat{\mathbf{H}}^{(\text {cor })}=\frac{1}{\|\mathbf{p}\|_{2}^{2}} \tilde{\mathbf{P}}_{i}^{\mathrm{H}} \mathbf{Y}_{i}
$$

Here, $\frac{1}{\|\mathbf{p}\|_{2}^{2}}$ is the energy of the pilot sequence transmitted at one transmit antenna (we implicitly assume that the energy of the pilot sequences do not change over time and that it is the same for all antennas). This estimator in fact corresponds to a simple correlator with a subsequent energy scaling.

\section{Tap-wise LMMSE Estimator}

A common problem of LMMSE channel estimation is that the autocorrelation matrix of the receive signal (which depends on the autocorrelation matrices of the channel and the noise) is unknown and has to be estimated. Especially in time dispersive MIMO channels, the full autocorrelation matrix becomes huge and very hard to estimate with high accuracy. Neglecting parts of this matrix reduces the complexity of the estimator but also sacrifices performance.

We tackle the problem of the huge autocorrelation matrices with the estimator structure illustrated in Figure 1. Here, the receive signal is first descrambled with delayed versions of the scrambling sequence $\mathbf{S}_{i}$ of the basestation. The descrambling operation acts as a whitening or decorrelation filter. The output signal of the descrambler is therefore only spatially correlated. As will be shown in the following, this structure greatly reduces the size of the autocorrelation matrices and allows for estimating the MIMO channel matrix at every delay individually. Hence, we call this estimator the tap-wise LMMSE channel estimator.

For the $m$-th channel tap $\left(0 \leq m \leq L_{\mathrm{h}}-1\right)$, the signal after 
the descrambling operation is given by

$$
\begin{aligned}
\mathbf{S}_{i-m}^{\mathrm{H}} \mathbf{Y}_{i}= & \sum_{k=0}^{L_{\mathrm{h}}-1} \mathbf{S}_{i-m}^{\mathrm{H}} \mathbf{X}_{i-k} \mathbf{H}_{k}+\mathbf{S}_{i-m}^{\mathrm{H}} \mathbf{N}_{i}= \\
= & \mathbf{P}_{i-m} \mathbf{H}_{m}+\mathbf{D}_{i-m} \mathbf{H}_{m}+ \\
& +\sum_{k \neq m} \mathbf{S}_{i-m}^{\mathrm{H}} \mathbf{X}_{i-k} \mathbf{H}_{k}+\mathbf{S}_{i-m}^{\mathrm{H}} \mathbf{N}_{i}
\end{aligned}
$$

This description can be reformulated by using the vec(.) operator

$$
\begin{aligned}
& \mathbf{r}_{i, m}=\left[\mathbf{r}_{i, m}^{(1) \mathrm{T}}, \ldots, \mathbf{r}_{i, m}^{\left(N_{\mathrm{R}}\right) \mathrm{T}}\right]^{\mathrm{T}}=\operatorname{vec}\left(\mathbf{S}_{i-m}^{\mathrm{H}} \mathbf{Y}_{i}\right)= \\
& =\underbrace{\left(\mathbf{I}_{N_{\mathrm{R}}} \otimes \mathbf{P}_{i-m}\right)}_{\mathbf{T}_{i-m}} \underbrace{\operatorname{vec}\left(\mathbf{H}_{m}\right)}_{\mathbf{h}_{m}}+ \\
& +\left(\mathbf{I}_{N_{\mathrm{R}}} \otimes \mathbf{D}_{i-m}\right) \underbrace{\operatorname{vec}\left(\mathbf{H}_{m}\right)}_{\mathbf{h}_{m}}+ \\
& +\sum_{k \neq m} \underbrace{\left(\mathbf{I}_{N_{\mathrm{R}}} \otimes\left(\mathbf{S}_{i-m}^{\mathrm{H}} \mathbf{X}_{i-k}\right)\right)}_{\tilde{\mathbf{X}}_{i-m, i-k}} \underbrace{\operatorname{vec}\left(\mathbf{H}_{k}\right)}_{\mathbf{h}_{k}}+ \\
& +\underbrace{\left(\mathbf{I}_{N_{\mathrm{R}}} \otimes \mathbf{S}_{i-m}^{\mathrm{H}}\right) \operatorname{vec}\left(\mathbf{N}_{i}\right)}_{\mathbf{w}_{i}}
\end{aligned}
$$

where $\otimes$ denotes the Kronecker product. The LMMSE estimator of the MIMO channel matrix at time delay $m$ is given by

$$
\hat{\mathbf{h}}_{m}=\mathbf{R}_{\mathbf{h}_{m} \mathbf{r}} \mathbf{R}_{\mathbf{r r}}^{-1} \mathbf{r}_{i, m} .
$$

The cross correlation $\mathbf{R}_{\mathbf{h}_{m} \mathbf{r}}$ is given by

$$
\begin{aligned}
\mathbf{R}_{\mathbf{h}_{m} \mathbf{r}} & =\mathrm{E}\left\{\mathbf{h}_{m} \mathbf{r}_{i, m}^{\mathrm{H}}\right\}=\mathrm{E}\left\{\mathbf{h}_{m} \mathbf{h}_{m}^{\mathrm{H}} \mathbf{T}_{i-m}^{\mathrm{H}}\right\}= \\
& =\mathbf{R}_{\mathbf{h}_{m} \mathbf{h}_{m}} \mathbf{T}_{i-m}^{\mathrm{H}},
\end{aligned}
$$

where we assumed $\mathrm{E}\left\{\mathbf{D}_{i-m}\right\}=\mathbf{0}$ and $\mathrm{E}\left\{\mathbf{S}_{i-m}\right\}=\mathbf{0}$.

For the following derivation, we assume that the time correlation of the channel coefficients is zero between different delays $m$, i.e. $\mathrm{E}\left\{\mathbf{h}_{m} \mathbf{h}_{k}^{\mathrm{H}}\right\}=\mathbf{0}$ for $k \neq m$. This assumption will lead to a very intuitive approximation of $\mathbf{R}_{\mathbf{r r}}$. The autocorrelation matrix of the receive signal is thus given by

$$
\begin{aligned}
\mathbf{R}_{\mathbf{r r}}= & \mathrm{E}\left\{\mathbf{r}_{i, m} \mathbf{r}_{i, m}^{\mathrm{H}}\right\}= \\
= & \mathbf{T}_{i-m} \mathbf{R}_{\mathbf{h}_{m} \mathbf{h}_{m}} \mathbf{T}_{i-m}^{\mathrm{H}}+ \\
& +\mathrm{E}\left\{\left(\mathbf{I}_{N_{\mathrm{R}}} \otimes \mathbf{D}_{i-m}\right) \mathbf{h}_{m} \mathbf{h}_{m}^{\mathrm{H}}\left(\mathbf{I}_{N_{\mathrm{R}}} \otimes \mathbf{D}_{i-m}^{\mathrm{H}}\right)\right\}+ \\
& +\sum_{k \neq m} \mathrm{E}\left\{\tilde{\mathbf{X}}_{i-m, i-k} \mathbf{h}_{k} \mathbf{h}_{k}^{\mathrm{H}} \tilde{\mathbf{X}}_{i-m, i-k}^{\mathrm{H}}\right\}+ \\
& +\mathbf{R}_{\mathbf{w w}} .
\end{aligned}
$$

This expression can be simplified using the following theorem.

Lemma 1: If the matrix $\mathbf{A}$ and the vector $\mathbf{b}$ show the structure

$$
\mathbf{A}=\left[\begin{array}{ccc}
a^{(1,1)} & \ldots & a^{\left(1, N_{\mathrm{b}}\right)} \\
\vdots & \ddots & \vdots \\
a^{\left(N_{\mathrm{a}}, 1\right)} & \ldots & a^{\left(N_{\mathrm{a}}, N_{\mathrm{b}}\right)}
\end{array}\right], \quad \mathbf{b}=\left[\begin{array}{c}
b^{(1)} \\
\vdots \\
b^{\left(N_{\mathrm{b}}\right)}
\end{array}\right],
$$

the elements of $\mathbf{A}$ are uncorrelated and identically distributed with zero mean and variance $\sigma_{a}^{2}$, the elements of $\mathbf{b}$ are identically distributed (not necessarily uncorrelated) with zero mean and variance $\sigma_{b}^{2}$, and the elements of $\mathbf{A}$ and $\mathbf{b}$ are statistically independent, we have

$$
\mathrm{E}\left\{\left(\mathbf{I}_{N_{\mathrm{d}}} \otimes \mathbf{A}\right) \mathbf{b b}^{\mathrm{H}}\left(\mathbf{I}_{N_{\mathrm{d}}} \otimes \mathbf{A}^{\mathrm{H}}\right)^{\mathrm{H}}\right\}=N_{\mathrm{b}} \sigma_{a}^{2} \sigma_{b}^{2} \mathbf{I}_{N_{\mathrm{a}} N_{\mathrm{d}}} .
$$

The proof of this expression is carried out by first showing

$$
\mathrm{E}\left\{\mathbf{A} \mathbf{b b}^{\mathrm{H}} \mathbf{A}^{\mathrm{H}}\right\}=N_{\mathrm{b}} \sigma_{a}^{2} \sigma_{b}^{2} \mathbf{I}_{N_{\mathrm{a}}}
$$

which itself can be shown by simple inspection of the matrixvector multiplication.

Lemma 1 can be used to simplify Equation (17) if we assume that the transmit sequences $\mathbf{d}_{i}$ and $\mathbf{x}_{i}$ are spatially and temporally uncorrelated. If we furthermore assume that the additive noise is white with variance $\sigma_{w}^{2}$ we obtain

$$
\begin{aligned}
\mathbf{R}_{\mathbf{r r}}= & \mathbf{T}_{i-m} \mathbf{R}_{\mathbf{h}_{m} \mathbf{h}_{m}} \mathbf{T}_{i-m}^{\mathrm{H}}+ \\
& +N_{\mathrm{T}} \sigma_{d}^{2} \sigma_{h_{m}}^{2} \mathbf{I}_{N_{\mathrm{c}} N_{\mathrm{R}}}+ \\
& +N_{\mathrm{T}} \sigma_{x}^{2} \sum_{k \neq m} \sigma_{h_{k}}^{2} \mathbf{I}_{N_{\mathrm{c}} N_{\mathrm{R}}}+ \\
& +\sigma_{w}^{2} \mathbf{I}_{N_{\mathrm{c}} N_{\mathrm{R}}} .
\end{aligned}
$$

The first term in (21) corresponds to the received energy of the pilot signals arriving at delay $m$ at the receiver. The three remaining terms in (21) almost add up to the total receive energy. The difference to the total receive energy is only given by the pilot energy which is usually low (about $10 \%$ of the total base station transmit energy). We therefore replace them by a diagonal matrix with the receive energy on the main diagonal,

$$
\begin{aligned}
\mathbf{R}_{\mathbf{r r}} & \approx \mathbf{T}_{i-m} \mathbf{R}_{\mathbf{h}_{m} \mathbf{h}_{m}} \mathbf{T}_{i-m}^{\mathrm{H}}+ \\
& +\underbrace{\sigma_{r}^{2} \mathbf{I}_{N_{\mathrm{R}}}}_{=\mathbf{R}_{\sigma_{r}}} \otimes \mathbf{I}_{N_{\mathrm{c}}} .
\end{aligned}
$$

Here, $\sigma_{r}^{2}$ corresponds to the average receive power per antenna. The first term in (22) is composed of the pilot signals and the channel correlation matrix at time delay $m$. The second term is approximated by the total received signal power. Using this approximation, the LMMSE estimator for the channel coefficients at delay $m$ is given by

$$
\begin{aligned}
\hat{\mathbf{h}}_{m}= & \mathbf{R}_{\mathbf{h}_{m} \mathbf{r}} \mathbf{R}_{\mathbf{r r}}^{-1} \mathbf{r}_{i, m}= \\
= & \mathbf{R}_{\mathbf{h}_{m} \mathbf{h}_{m}} \mathbf{T}_{i-m}^{\mathrm{H}} . \\
& \cdot\left[\mathbf{T}_{i-m} \mathbf{R}_{\mathbf{h}_{m} \mathbf{h}_{m}} \mathbf{T}_{i-m}^{\mathrm{H}}+\mathbf{R}_{\sigma_{r}} \otimes \mathbf{I}_{N_{c}}\right]^{-1} \mathbf{r}_{i, m} .
\end{aligned}
$$

Applying the Woodbury identity [7] for positive definite matrices $\mathbf{P}$ and $\mathbf{R}$

$$
\mathbf{P B}^{\mathrm{H}}\left(\mathbf{B P} \mathbf{B}^{\mathrm{H}}+\mathbf{R}\right)^{-1}=\left(\mathbf{P}^{-1}+\mathbf{B}^{\mathrm{H}} \mathbf{R}^{-1} \mathbf{B}\right)^{-1} \mathbf{B}^{\mathrm{H}} \mathbf{R}^{-1},
$$


and the relation $(\mathbf{A} \otimes \mathbf{B})(\mathbf{C} \otimes \mathbf{D})=\mathbf{A C} \otimes \mathbf{B D}$, the LMMSE estimator can be reformulated to

$$
\begin{aligned}
\hat{\mathbf{h}}_{m}= & {\left[\mathbf{R}_{\mathbf{h}_{m} \mathbf{h}_{m}}^{-1}+\mathbf{T}_{i-m}^{\mathrm{H}}\left(\mathbf{R}_{\sigma_{r}}^{-1} \otimes \mathbf{I}_{N_{c}}\right) \mathbf{T}_{i-m}\right]^{-1} . } \\
& \cdot \mathbf{T}_{i-m}^{\mathrm{H}}\left(\mathbf{R}_{\sigma_{r}}^{-1} \otimes \mathbf{I}_{N_{\mathrm{c}}}\right) \mathbf{r}_{i, m}= \\
= & {\left[\mathbf{R}_{\mathbf{h}_{m} \mathbf{h}_{m}}^{-1}+\left(\mathbf{R}_{\sigma_{r}}^{-1} \otimes \mathbf{P}_{i-m}^{\mathrm{H}} \mathbf{P}_{i-m}\right)\right]^{-1} . } \\
& \cdot\left(\mathbf{R}_{\sigma_{r}}^{-1} \otimes \mathbf{P}_{i-m}^{\mathrm{H}}\right) \mathbf{r}_{i, m} .
\end{aligned}
$$

However, this LMMSE estimator requires the knowledge of the channel autocorrelation matrix $\mathbf{R}_{\mathbf{h}_{m} \mathbf{h}_{m}}$, which is usually not known. In our simulations we will consider the case of a perfectly known channel autocorrelation matrix and the case of "instantaneously" (similar to an approach for OFDM channel estimation presented in [8]) estimating this matrix. Note that any channel estimator utilizing an improved estimate of the channel autocorrelation will show a performance between those two cases.

\section{Instantaneous channel autocorrelation estimation}

The LMMSE channel estimator using the instantaneous channel auto correlation can be described by the following threestage process:

1) Perform a correlation based channel estimation

$$
\hat{\mathbf{h}}_{m}^{(\text {cor })}=\frac{1}{\|\mathbf{p}\|_{2}^{2}}\left(\mathbf{I}_{N_{\mathrm{R}}} \otimes \mathbf{P}_{i-m}^{\mathrm{H}}\right) \mathbf{r}_{i, m}
$$

with $\|\mathbf{p}\|_{2}^{2}=\left\|\mathbf{p}^{\left(n_{\mathrm{t}}\right)}\right\|_{2}^{2}$ (assuming that the pilot power is equally distributed over the transmit antennas).

2) Calculate the channel gains, and assume them to be spatially uncorrelated. Here, we use the notation $(.)_{n}$ for denoting the $n$-th element of a vector.

$$
\hat{\mathbf{R}}_{\mathbf{h}_{m} \mathbf{h}_{m}}^{-1}=\operatorname{diag}\left(\frac{1}{\left|\left(\hat{\mathbf{h}}_{m}^{(\text {cor })}\right)_{n}\right|^{2}}\right) \quad ; n=1, \ldots, N_{\mathrm{R}} N_{\mathrm{T}} .
$$

3) Use the estimate of $\mathbf{R}_{\mathbf{h}_{m} \mathbf{h}_{m}}^{-1}$ in the equation for the LMMSE estimate to improve the correlation based channel estimate

$$
\begin{aligned}
\hat{\mathbf{h}}_{m}^{\text {LMMSE })}= & {\left[\hat{\mathbf{R}}_{\mathbf{h}_{m} \mathbf{h}_{m}}^{-1}+\mathbf{R}_{\sigma_{r}}^{-1} \otimes\left(\mathbf{P}_{i-m}^{\mathrm{H}} \mathbf{P}_{i-m}\right)\right]^{-1} } \\
& \cdot\|\mathbf{p}\|^{2}\left(\mathbf{R}_{\sigma_{r}}^{-1} \otimes \mathbf{I}_{N_{\mathrm{T}}}\right) \hat{\mathbf{h}}_{m}^{\text {(cor) }} .
\end{aligned}
$$

At the cost of higher complexity, the LMMSE channel estimate obtained in Step 3 can be used to improve the estimate of the channel autocorrelation matrix $\hat{\mathbf{R}}_{\mathbf{h}_{m}} \mathbf{h}_{m}$ in Step 2. Subsequently, the channel estimate can be improved again. Although we will not consider such an iterative channel estimator structure in the following, first simulation results showed that the channel estimator MSE can be further decreased compared to a non-iterative structure.

\section{Complexity Considerations}

The computational complexity of the tap-wise LMMSE channel estimator with instantaneous autocorrelation estimation is slightly increased compared to the LS channel estimator. The first step of both both estimators - the calculation of the
TABLE I

SIMULATION PARAMETERS.

\begin{tabular}{|l|c|}
\hline Parameter & Value \\
\hline \hline Number of active users & 4 \\
Desired user CQI & 13 \\
Modulation alphabet & 4 -QAM \\
Coding rate & 0.6 \\
Interfering HS-PDSCH $E_{\mathrm{c}} / I_{\mathrm{or}}$ & {$[-6,-8,-10] \mathrm{dB}$} \\
Interfering user CQIs & {$[16,11,8]$} \\
Precoding coeff. of interf. users & {$[1,-j],[1, j],[1,-1]$} \\
CPICH $E_{\mathrm{c}} / I_{\mathrm{or}}$ & $-10 \mathrm{~dB}$ \\
SCH/PCCPCH $E_{\mathrm{c}} / I_{\mathrm{or}}$ & $-12 \mathrm{~dB}$ \\
User equipment capability & 6 \\
Channel model & 22 \\
$L_{\mathrm{h}}$ & 40 \\
$L_{\mathrm{f}}$ & $3 \mathrm{~km} / \mathrm{h}$ \\
UE speed & $15 \mathrm{~dB}$ \\
$I_{\mathrm{or}} / I_{\mathrm{oc}}$ & off \\
OCNS & uncorrelated Pedestrian B $[9]$ \\
Receiver & interference aware equalizer $[10,11]$ \\
\hline
\end{tabular}

correlation-based channel estimate-is exactly the same. In the second step, $N_{\mathrm{R}}$ matrix inverses of dimension $N_{\mathrm{T}} \times N_{\mathrm{T}}$ have to be calculated for every channel tap. The increased complexity of the tap-wise LMMSE channel estimator basically comes from the multiple descrambling operations and the estimation of the channel autocorrelation matrix like, e.g., in (27).

\section{Simulation Results}

In this section, link level simulation results for a TxAA HSDPA system are presented. We simulate the experienced data throughput of one user (the "desired" user in the following) in a fully loaded cell, i.e., strong interference is generated by the data channels assigned to three other users. We assume that the three other users are at a constant position in the cell, i.e., their channel stays constant. Therefore, the $E_{\mathrm{c}} / I_{\mathrm{or}}$ (the ratio defining the amount of the total basestation power allocated to these users) values as well as the precoding coefficients of the other users are assumed to be fixed. The precoding coefficients of the desired user are adaptively adjusted, as defined for TxAA [3]. In the simulations we employ a low complexity interference aware LMMSE equalizer $[10,11]$ in contrast to optimum MIMO detection algorithms [12]. Our simulation settings are shown in detail in Table I.

Figure 2 and 3 show the simulated data throughput over an uncorrelated Pedestrian B channel for a $2 \times 1$ and a $2 \times 2$ system, respectively. The LS estimator shows only slightly better performance (about $0.2 \mathrm{~dB}$ ) than the correlation based estimator since it only accounts for the imperfect orthogonality of the training sequences (the factor $\left(\tilde{\mathbf{P}}_{i}^{\mathrm{H}} \tilde{\mathbf{P}}_{i}\right)^{-1}$ in (11)) and it does not consider interference at all. The tap-wise LMMSE with the simplest possible (instantaneous) channel autocorrelation estimation already outperforms the correlation based estimator by about $0.5 \mathrm{~dB}$ in the $2 \times 1$ and $0.85 \mathrm{~dB}$ in the $2 \times 2$ system. If a better channel autocorrelation estimator is implemented these gains can be increased to $0.8 \mathrm{~dB}$ and $1.35 \mathrm{~dB}$, respectively. Table II summarizes the gains of the various channel estimators over the correlation-based estimator. 


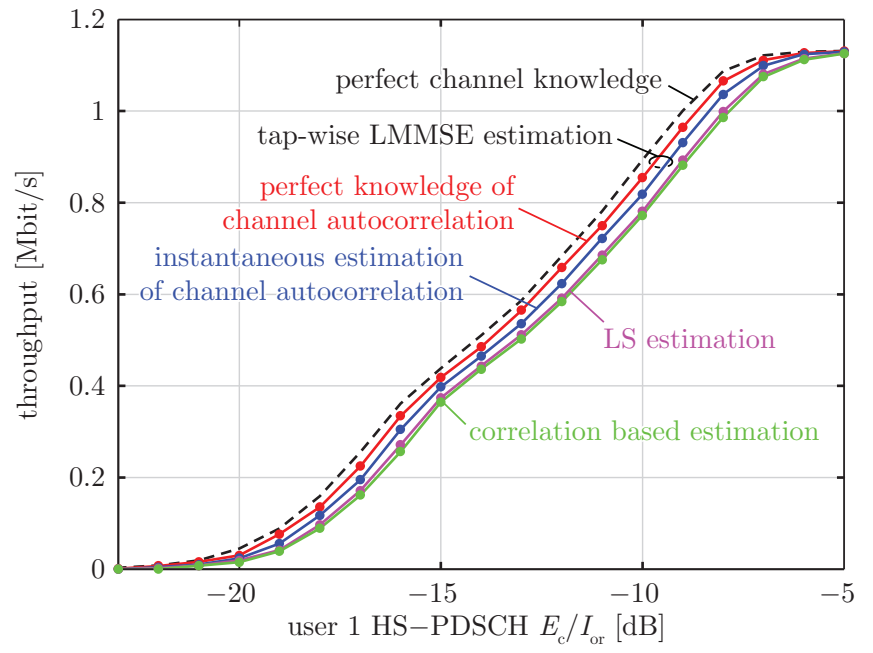

Fig. 2. Throughput of User 1 in a $2 \times 1$ Pedestrian B TxAA multiuser scenario.

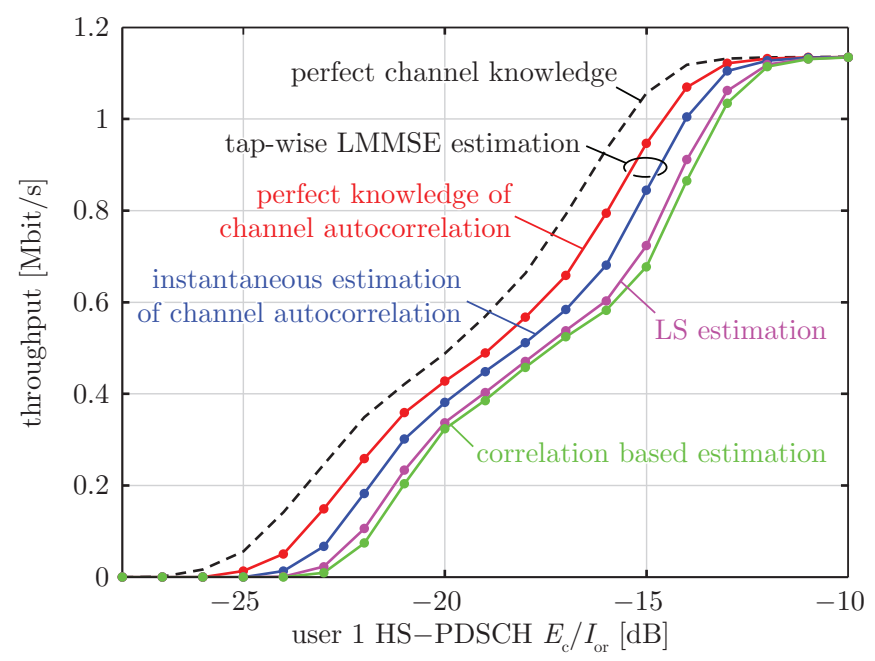

Fig. 3. Throughput of User 1 in a $2 \times 2$ Pedestrian B TxAA multiuser scenario.

The gains are given at $90 \%$ of the maximum throughput for the simulated CQI value, since [3] specifies that the mobile equipment should adjust its reported CQI value in order to achieve a $10 \%$ block error ratio.

TABLE II

SNR GAIN OVER THE CORRELATION BASED ESTIMATOR (AT $90 \%$ OF THE MAXIMUM THROUGHPUT)

\begin{tabular}{|l|r|r|}
\hline Channel estimator & $2 \times 1$ & $2 \times 2$ \\
\hline \hline Perfect & $1.15 \mathrm{~dB}$ & $2.25 \mathrm{~dB}$ \\
Tap-wise LMMSE, PDP perf. & $0.8 \mathrm{~dB}$ & $1.35 \mathrm{~dB}$ \\
Tap-wise LMMSE, PDP inst. & $0.5 \mathrm{~dB}$ & $0.85 \mathrm{~dB}$ \\
LS estimator & $0.15 \mathrm{~dB}$ & $0.2 \mathrm{~dB}$ \\
correlation-based & $0.0 \mathrm{~dB}$ & $0.0 \mathrm{~dB}$ \\
\hline
\end{tabular}

\section{Conclusions}

The tap-wise LMMSE channel estimator shows good performance while the complexity is only slightly increased compared to the LS channel estimator. Depending on the type of channel autocorrelation estimation and the interference situation of the receiver, the performance gain over the correlation-based channel estimator is between 0.85 and $1.35 \mathrm{~dB}$ in the simulated scenario. The complexity increase over the LS estimator is mainly caused by the multiple descrambling operations (one descrambler for every tap that has to be estimated, just as in a standard RAKE receiver) and the estimator for the channel autocorrelation function. In future work, an iterative estimator structure that alternately improves the accuracy of the channel estimate and the channel autocorrelation estimate will be investigated.

\section{ACKNOWLEDGMENT}

This work has been funded by the Christian Doppler Laboratory for Design Methodology of Signal Processing Algorithms, the Austrian Research Centers $\mathrm{GmbH}$, as well as the $\mathrm{ftw}$. through the $\mathrm{C} 12$ project. The authors would like to thank Martin Wrulich for proofreading the paper and valuable suggestions for improvements.

\section{REFERENCES}

[1] 3GPP, "Technical specification group radio access network; Multiple-Input Multiple Output in UTRA," 3GPP, Tech. Rep. 25.876 V7.0.0, Mar. 2007. [Online]. Available: http://www.3gpp.org/ftp/Specs/html-info/25876.htm

[2] R. Tresch, C. Mehlführer, and M. Guillaud, "LMMSE channel estimation for MIMO W-CDMA with out-of-cell interference mitigation," in Conference Record of the Fourtysecond Asilomar Conference on Signals, Systems and Computers, 2008 , Pacific Grove, CA, USA, Oct. 2008.

[3] 3GPP, "Technical specification group radio access network; physical layer procedures (FDD) (Tech. Spec. 25.214 V3.0.0)," 1999. [Online]. Available: http://www.3gpp.org/ftp/Specs/archive/25_series/25.214/

[4] _ " "Technical specification group radio access network; physical layer - general description (FDD) (Tech. Spec. 25.201 V5.3.0)," June 2005. [Online]. Available: http://www.3gpp.org/ftp/Specs/html-info/25201.htm

[5] B. Lindoff, C. Östberg, and H. Eriksson, "Channel estimation for the W-CDMA system, performance and robustness analysis from a terminal perspective," in Proc. IEEE 49th Vehicular Technology Conference (VTC 1999), vol. 2, July 1999, pp. 1565-1569. [Online]. Available: http://ieeexplore.ieee.org/iel5/6314/ 16887/00780649.pdf?tp=\&arnumber $=780649$

[6] S. McBeath, M. Ahmed, and K. Rohani, "Impact of imperfect estimators on W-CDMA receiver performance with MIMO antenna systems," in Proc. IEEE 58th Vehicular Technology Conference (VTC 2003-Fall), vol. 2, Oct. 2003, pp. 1152-1156. [Online]. Available: http://ieeexplore.ieee.org/iel5/9004/28569/ 01285202.pdf?tp $=$ \&arnumber $=1285202$

[7] T. K. Moon and W. C. Stirling, Mathematical Methods and Algorithms for Signal processing, 1st ed. Prentice Hall, 2000.

[8] C. Mehlführer, S. Caban, and M. Rupp, "An accurate and low complex channel estimator for OFDM WiMAX," in Proc. Third International Symposium on Communications, Control, and Signal Processing (ISCCSP 2008), St. Julians, Malta, Mar. 2008, pp. 922-926. [Online]. Available: http://publik.tuwien.ac.at/ files/pub-et_13650.pdf

[9] "Recommendation ITU-R M.1225: Guidelines for evaluation of radio transmission technologies for IMT-2000," Tech. Rep., 1997.

[10] C. Mehlführer, M. Wrulich, and M. Rupp, "Intra-cell interference aware equalization for TxAA HSDPA," in Proc. IEEE International Symposium on Wireless Pervasive Computing (ISWPC 2008), Santorini, Greece, May 2008, pp. 406-409. [Online]. Available: http://publik.tuwien.ac.at/files/pub-et_13749.pdf

[11] M. Wrulich, C. Mehlführer, and M. Rupp, "Interference aware MMSE equalization for MIMO TxAA," in Proc. Third International Symposium on Communications, Control, and Signal Processing (ISCCSP 2008), St. Julians, Malta, Mar. 2008, pp. 1585-1589. [Online]. Available: http://publik.tuwien.ac.at/files/pub-et_13657.pdf

[12] M. Rupp, M. Guillaud, and S. Das, "On MIMO decoding algorithms for UMTS," in Conference Record of the Thirty-Fifth Asilomar Conference on Signals, Systems and Computers, 2001, vol. 2, Nov. 2001, pp. 975-979. [Online]. Available: http://ieeexplore.ieee.org/iel5/7744/21273/00987640.pdf?tp= \&isnumber $=$ \&arnumber $=987640$ 


\section{Reference:}

C. Mehlführer and M. Rupp, "Novel Tap-wise LMMSE Channel Estimation for MIMO W-CDMA," in Proc. 51st Annual IEEE Globecom Conference, 2008, New Orleans, LA, USA, Nov. 2008. [Online].

Available: http://publik.tuwien.ac.at/files/PubDat_169129.pdf

BibTeX:

a InProceedings $\{$ Globecom 2008 ,

author $=\quad$ Christian Mehlf $\backslash$ "uhrer and Markus Rupp\},

title $=\quad\{$ Novel Tap-wise $\{$ LMMSE $\}$ Channel Estimation for \{MIMO W-CDMA $\}$

booktitle $=\{$ Proc. 51st Annual IEEE Globecom Conference, 2008$\}$

month $=$ nov,

year $=\quad 2008$,

address $=\{$ New Orleans, LA, USA

url =

\{http://publik.tuwien.ac.at/files/PubDat_169129.pdf 